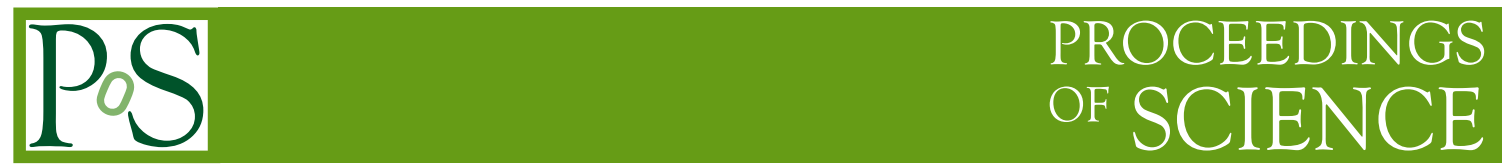

\title{
The identification of glueballs - further tests
}

\author{
Wolfgang Ochs* \\ Max-Planck-Institut fuer Physik, Foehringer Ring 6, D-80805 Munich, Germany \\ E-mail: och. @mpp.mpg. de
}

\begin{abstract}
The indirect evidence for gluonic objects in gluon jets is recalled, more accurate tests at the LHC are possible. Estimates of gluonic and quarkonic components of scalar mesons are presented, which could be improved by further studies, in particular of $B$ and $B_{S}$ decays. The results are consistent with the scalar glueball mixing into $f_{0}(500)$ and $f_{0}(1500)$. The study of symmetry relations for 2-body decay rates of charmonium $\chi_{c}$ is suggested as crucial test in the identification of $q \bar{q}$ multiplets and glueballs. Further related results are discussed in the recent review [1].
\end{abstract}

XV International Conference on Hadron Spectroscopy-Hadron 2013

4-8 November 2013

Nara, Japan

${ }^{*}$ Speaker. 


\section{QCD expectations for glueballs}

The identification of glueballs remains a challenge in meson spectroscopy ever since they have been discussed within QCD as bound states of the self-interacting gluons [2]. Today, on the theory side, lattice QCD aims at predictions from basic principles. There are converging results within the theory of gluons only ("quenched") of a spectrum of $g g$ bound states, "gluonium", with the lightest state being a scalar $J^{P C}=0^{++}$of mass $1500-1700 \mathrm{MeV}$. In full QCD the gluonium state can mix with $q \bar{q}$ states. For the lightest scalar flavour singlet state there are conflicting results: most recently the full ("unquenched") calculation yields comparable results to the purely gluonic calculation [3] while earlier studies suggested the lightest states are of mass around 1000 and $1500 \mathrm{MeV}$ and they are mixed from $q \bar{q}$ and $g g$ components [4].

An alternative path to spectroscopy is based on QCD sum rules which include some phenomenological parameters. Already long ago the lightest gluonium states have been located near $1000 \mathrm{MeV}$ and $1500 \mathrm{MeV}$ [5]. A more recent analysis finds that the states at these two masses should be viewed as mixtures of $q \bar{q}$ and $g g$ components [6].

A commonly accepted view of these QCD results is still missing.

\section{Indications of glueball production in gluon jets}

While there is a clear evidence for glueballs in the theory of gluons, the evidence on the experimental side is still controversial; in particular, there are very different interpretations of the scalar meson spectrum. However, there is a remarkable effect which strongly points towards the existence of gluonic objects. In a well known phenomenology of "quark fragmentation" the most energetic hadrons are those which carry a valence quark in common with the initial quark of the jet, for example, the $\pi^{+}$in a $u$-quark jet. In analogy one can hypothesize that glueballs are the leading objects in a gluon jet, an idea with a long history [7]. As a practical tool one may isolate leading clusters in jets by a rapidity gap: while in quark jets the charge of the leading $q \bar{q}$ object is $Q_{q}=0, \pm 1$ the corresponding charge of $(g g)$ in a gluon jet should be $Q_{g}=0$ [8]. Remarkably, all the studies at LEP by the experiments ALEPH, DELPHI and OPAL [9] have shown consistently, that the "standard" Monte Carlo JETSET without glueballs included is able to reproduce the charge properties of quark jets but failed to describe the charge distribution of leading clusters in gluon jets: there was an excess of neutral charge $Q_{g}$. An example is shown in figure 1 for the ALEPH Collaboration with an excess at $Q_{g}=0$ of about $40 \%$. Such an effect is just what is expected for glueballs, so we take these observations as an indirect evidence for gluonic objects. Alternatively, the MC program has modeled the gluon jet incorrectly.

In any case it would be desirable to clarify this issue at the LHC $[8,1]$. Here the higher jet energy allows for larger rapidity gaps and there is a high production rate of gluon jets to be compared with quark jets. Alternatively, one may compare the Feynman x-distribution of a resonance with composition in doubt (example $f_{0}(1500)$ ) with a known "test state" (examples $\rho(770), f_{2}(1270)$, $\phi(1020))$. In a quark $u, d$ jet the leading $\rho$ would be "harder" than a glueball, whereas in a gluon jet the behaviour should be opposite. 


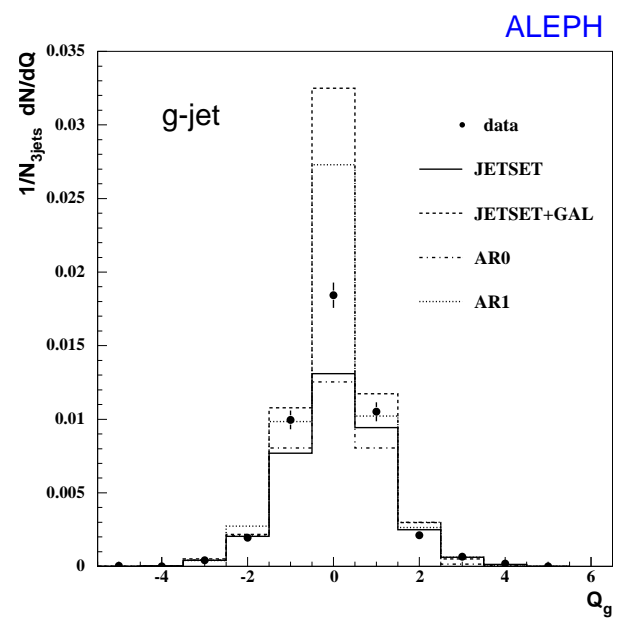

Figure 1: Distribution of the charge $Q_{g}$ of the leading cluster in a gluon jet beyond a rapidity gap $\Delta y=1.5$ showing a surplus of events at $Q_{g}=0$ over the JETSET Monte Carlo prediction (full line) by $40 \%$.

\section{Scalar meson spectroscopy with glueballs}

Most searches for glueballs have concentrated on the scalar sector as the lightest glueball is a scalar according to QCD. A scalar glueball could mix into the following states listed in [10]

$$
f_{0}(500), f_{0}(980), f_{0}(1370), f_{0}(1500), f_{0}(1710) \ldots
$$

and there are various such mixing schemes. According to an early suggestion the heaviest three states in (3.1) are mixed from the two members of a $q \bar{q}$ nonet and a glueball centered around 1500 $\mathrm{MeV}$ [11]. Then there could be another nonet including the two $f_{0}$ states below $1 \mathrm{GeV}$ together with $a_{0}(980)$ and the $K_{0}^{*}(900) / \kappa$ particle. A problem here is with $f_{0}(1370)$ whose existence is in doubt as there is no evidence found in the uniquely identified energy-independent phase shift solutions (for a recent review of the studied channels, see [1]); also the situation with $\kappa$ is difficult to clarify $[10,1]$. According to another suggestion the $q \bar{q}$ nonet includes $f_{0}(980)$ and $f_{0}(1500)$ while the broad $f_{0}(500)$ with "Breit-Wigner mass" $m_{B W} \sim 1000 \mathrm{MeV}$, where the phase shifts pass $90^{\circ}$, is related to the glueball [12], related works in [5, 13, 14].

In [1] an attempt has been made to determine the gluonic component as given by the gluonic mixing angle $\phi_{G}$ and the flavour mixing angle $\phi_{s c}$ of scalars from experimental data, for example

$$
\left|f_{0}(1500)\right\rangle=\cos \phi_{G}|q \bar{q}\rangle+\sin \phi_{G}|g g\rangle ; \quad|q \bar{q}\rangle=\cos \phi_{s c}|n \bar{n}\rangle-\sin \phi_{s c}|s \bar{\zeta}\rangle .
$$

The following results have been obtained:

- $f_{0}(980)$ ): the scalar mixing angle (defined as in [1]) is found as $\phi_{s c}=(30 \pm 3)^{\circ}$ (similar to $\eta^{\prime}$ with $\left.\phi_{p s} \sim 42^{\circ}\right)$ from $\pi \pi \rightarrow \pi \pi / K \bar{K} ; J / \psi \rightarrow(\omega / \phi) \pi \pi, D_{s} \rightarrow 3 \pi, \gamma \gamma \rightarrow f_{0}(980) / a_{0}(980)$; there is no definitive evidence for a gluonic contribution $\left(\cos ^{2} \phi_{G} \approx 0.75 \pm 0.25\right)$.

- $f_{0}(500)$ : couplings $r_{K \pi} \equiv g_{K \bar{K}}^{2} / g_{\pi \pi}^{2}=0.3-0.6$ and $r_{\eta \pi} \equiv g_{\eta \eta}^{2} / g_{\pi \pi}^{2} \sim 0.2$ based on model fits to $\pi \pi \rightarrow \pi \pi / K \bar{K} / 4 \pi / \eta \eta$ amplitudes $[15,16]$ suggest this state to be neither pure glue $\left(r_{K \pi}=4 / 3\right)$ nor pure nonstrange $q q \bar{q} \bar{q}\left(r_{K \pi}=0\right)$. 
- $f_{0}(1500)$ : the 2-body branching ratios in [10] do not fix $\phi_{s c}$; given $\phi_{s c}$ the gluon component $r_{G}=r_{G}^{0} \tan \phi_{G}$ is fixed where $r_{G}^{0}=G_{0} / g_{0}$ refers to decay couplings $G_{0}, g_{0}$ of $(g g)$ and $(q \bar{q})$.

In a minimal mixing scheme [1] one assumes $f_{0}(980)$ without glue and with $\phi_{s c}=30^{\circ}$, the glueball is mixed into $f_{0}(1500)$ and $f_{0}(500)$ with

$$
\left|f_{0}(500)\right\rangle=\sin \phi_{G}|q \bar{q}\rangle-\cos \phi_{G}|g g\rangle ; \quad|q \bar{q}\rangle=\cos \phi_{s c}|n \bar{n}\rangle-\sin \phi_{s c}|s \bar{s}\rangle .
$$

With the above experimental results for $f_{0}(500)$ and $f_{0}(1500)$ we obtain

$$
\phi_{G} \sim 50^{\circ}, \quad r_{G}^{0} \approx 0.5
$$

i.e. a strongly mixed system. Note that for $\phi_{G}=0$ we recover the model [12], while the composition of $f_{0}(1500)$ is similar to the model [11] but with a different $q \bar{q}$ multiplet. An interesting aspect of this mixing scheme is the compatibility with certain results obtained from lattice QCD and from QCD sum rules (see section 1).

Further tests on the flavour composition are possible from the decays $B \rightarrow J / \psi f_{0}$ and $B_{s} \rightarrow$ $J / \psi f_{0}$ which allow for an isolation of the $d \bar{d}$ and the $s \bar{s}$ components of $f_{0}$ respectively. From $B_{S}$ decays using equations $(3.2,3.3)$ one can obtain the ratio of the $s \bar{s}$ components as

$$
r_{f_{0}^{\prime} / f_{0}}^{s}=\frac{p^{3} B\left(\bar{B}_{s}^{0} \rightarrow J / \psi f_{0}(1500)\right)}{\left(p^{\prime}\right)^{3} B\left(\bar{B}_{s}^{0} \rightarrow J / \psi f_{0}(980)\right)}, \quad r_{f_{0}^{\prime} / f_{0}}^{s}=\cos ^{2} \phi_{G}^{f_{0}^{\prime}} \tan ^{2} \phi_{s c}
$$

with $p\left(p^{\prime}\right)$ the respective momenta in the $B_{s} \mathrm{cms}$. The LHCb results for $f_{0} \rightarrow \pi \pi$ [17] yield two solutions of the phase shift analysis, from the recent data on the $f_{0} \rightarrow K^{+} K^{-}$decay mode [18] we conclude that the "alternate solution" is preferred with $r_{f_{0}^{\prime} / f_{0}}^{s}=0.15 \pm 0.05$; this supersedes our choice in [1]. Then we derive from (3.5) using $\phi_{s c}=30^{\circ}$ the gluonic mixing angle $\phi_{G}^{f_{0}^{\prime}}=48^{\circ} \pm 10^{\circ}$ in good agreement with our result above in (3.4).

The flavour mixing angle $\phi_{s c}$ for a particular $f_{0}$ meson can be derived from the ratio of the $J / \psi f_{0}$ decays of $B$ and $B_{s}[1]$. First indications of a $f_{0}(1500)$ signal in $B_{d}$ decays become visible [19]. A measurement of these ratios for the light scalars $f_{0}$ would greatly advance our understanding of this sector. Note that $f_{0}(500)$ in our view is to be represented in a phase shift analysis as broad object centered at $m_{B W} \sim 1000 \mathrm{MeV}[12,1]$.

\section{Nonets and glueballs from symmetry relations}

Another area of promising research are the decay rates of charmonia (or bottomonia). In the decay $\chi_{c} \rightarrow g g$ the pairs of hadrons from the same $q \bar{q}$ multiplet form a flavour singlet state. For pairs of pseudoscalars one expects for the phase space corrected decay rates

$$
\gamma_{i j}^{2}\left(\chi_{c} \rightarrow \pi \pi: K \bar{K}: \eta \eta: \eta \eta^{\prime}: \eta^{\prime} \eta^{\prime}\right)=3: 4: 1: 0: 1
$$

reflecting the $S U(3)_{f l}$ symmetry. These relations work rather well for decays of $\chi_{c 0}\left(0^{++}\right)$and $\chi_{c 2}\left(0^{++}\right)$within $10-20 \%$ [1]. Correspondingly, one may test this symmetry for decays into members of other multiplets, in particular of the scalar multiplet. The relations in analogy to (4.1) are expected to fail if the wrong members of the multiplet are chosen, furthermore, deviations are 
expected for the isoscalar members in the presence of glueballs. The PDG results [10] (based on BES-II [20]) yield the ratio of decay rates after correction for phase space $q$ and charge weight $c$

$$
r_{f_{0} / K^{*}}^{\chi_{c 0}} \equiv \frac{B\left(\chi_{c 0}(3415) \rightarrow f_{0}(980) f_{0}(980)\right) /(c q)}{B\left(\chi_{c 0}(3415) \rightarrow K_{0}^{*}(1430) \bar{K}_{0}^{*}(1430)\right) /\left(c^{\prime} q^{\prime}\right)}, \quad r_{f_{0} / K^{*}}^{\chi_{c 0}}=1.64 \pm 0.73
$$

which is consistent with symmetry $\left(r_{f_{0} / K^{*}}^{\chi_{c 0}}=1\right)$ and so with $f_{0}(980)$ and $K_{0}^{*}(1430)$ being in the same $q \bar{q}$ nonet $[13,12]$. Higher accuracy and measurements of relations to other scalars like $a_{0}(980), f_{0}(1500)$ and also to $f_{0}(500), K_{0}^{*}(900) / \kappa$ could be decisive. The results also prefer $f_{0}(980)$ to be a $(q \bar{q})$ rather than a $(q q \bar{q} \bar{q})$ state according to [1] from a comparison with $p \bar{p}$ data.

\section{Summary}

QCD predicts the existence of glueballs; mass and mixing properties of the scalar glueball need further clarification. Experimental hints for glueballs come from the excess of neutral clusters in gluon jets. The intrinsic quark-gluon structure of mesons is accessible experimentally; the role of $B, B_{s}$ data is stressed. Tests of symmetry relations for $\chi_{c}$ decays help identifying $q \bar{q}$ nonets.

\section{References}

[1] W. Ochs, "The Status of Glueballs," J. Phys. G 40 (2013) 043001

[2] H. Fritzsch and P. Minkowski, Nuovo Cim. A 30 (1975) 393

[3] C. M. Richards et al. [UKQCD Collaboration], Phys. Rev. D 82 (2010) 034501

[4] A. Hart et al. [UKQCD Collaboration], Phys. Rev. D 74 (2006) 114504

[5] S. Narison and G. Veneziano, Int. J. Mod. Phys. A 4 (1989) 2751

[6] D. Harnett, R. T. Kleiv, K. Moats and T. G. Steele, Nucl. Phys. A 850 (2011) 110

[7] P. Roy and T. F. Walsh, Phys. Lett. B 78 (1978) 62

[8] P. Minkowski and W. Ochs, Phys. Lett. B 485 (2000) 139; XIV Int. Conf. on Hadron Specrtroscopy (Hadron 2011), 13-17 June 2011, Munich, Germany), arXiv:1108.0589 [hep-ph]

[9] S. Schael et al. [ALEPH Coll.], Eur. Phys. J. C 48 (2006) 685; J. Abdallah et al. [DELPHI Coll.], Phys. Lett. B 643 (2006) 147; G. Abbiendi et al. [OPAL Coll.], Eur. Phys. J. C 35 (2004) 293

[10] J. Beringer et al. (Particle Data Group), Phys. Rev. D 86 (2012) 010001

[11] C. Amsler and F. E. Close, Phys. Rev. D 53 (1996) 295

[12] P. Minkowski and W. Ochs, Eur. Phys. J. C 9 (1999) 283

[13] E. Klempt et al. Phys. Lett. B 361 (1995) 160; V. Dmitrasinovic, Phys. Rev. C 53 (1996) 1383

[14] V. V. Anisovich and A. V. Sarantsev, Eur. Phys. J. A 16 (2003) 229

[15] R. Kaminski, G. Mennessier and S. Narison, Phys. Lett. B 680 (2009) 148

[16] D. V. Bugg, Eur. Phys. J. C 47 (2006) 45

[17] R. Aaij et al. [LHCb Collaboration], Phys. Rev. D 86 (2012) 052006

[18] R. Aaij et al. [LHCb Collaboration], Phys. Rev. D 87 (2013) 072004

[19] R. Aaij et al. [LHCb Collaboration], Phys. Rev. D 87 (2013), 052001 and arXiv:1310.2145

[20] M. Ablikim et al. [BES Collaboration], Phys. Rev. D 72 (2005) 092002; D 70 (2004) 092002 